\title{
Correction to: Oxymatrine Inhibits Bocavirus MVC Replication, Reduces Viral Gene Expression and Decreases Apoptosis Induced by Viral Infection
}

\author{
Yanqin Ding ${ }^{1} \cdot \mathrm{Na}^{1}{ }^{1} \cdot$ Jinhan Sun $^{2} \cdot \operatorname{Linran}_{Z^{2}}$ Jang $^{1} \cdot$ Jianhui Guo $^{1} \cdot$ Xueqi Hao $^{3} \cdot$ Yuning Sun $^{1}$ (D)
}

Published online: 3 July 2019

(C) Wuhan Institute of Virology, CAS 2019

\section{Correction to: Virologica Sinica (2019) 34:78-87}

https://doi.org/10.1007/s12250-019-00088-2

In the original version of Fig. 2, the marker line between column 2 and column 6 is redundant (Fig. 2A), and MVC label and MVC/OMT label were accidentally

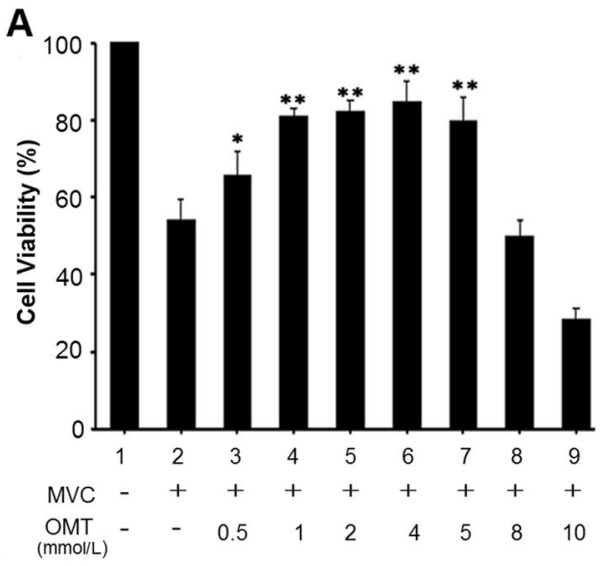

Fig. 2 OMT enhances the viability of MVC-infected cells. A Cells were infected with MVC (MOI:10) and treated with different concentrations $(0.5,1,2,4,5,8,10 \mathrm{mmol} / \mathrm{L})$ of OMT. B Cells were infected with MVC at different MOI values $(2.5,5,10,20,40,80)$ swapped (Fig. 2B). The correct Fig. 2 is provided below.

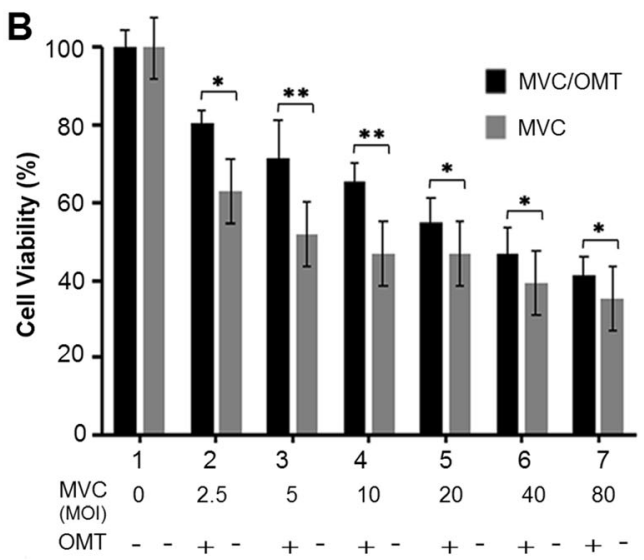

and treated with OMT ( $4 \mathrm{mmol} / \mathrm{L}$, the maximal safety concentration). After $72 \mathrm{~h}$, optical density (OD) was detected by CCK- 8 assay. The data are shown as mean \pm SD of three independent experiments. $* P<0.05 ; * * P<0.01$.
Yanqin Ding and Na Li have contributed equally to this publication.

The original article can be found online at https:// doi.org/10.1007/s12250-019-00088-2.

Yuning Sun

sunraining2008@hotmail.com

1 Department of Biochemistry and Molecular Biology, Key Laboratory of Fertility Preservation and Maintenance of Ministry of Education, School of Basic Medical Science,

Ningxia Medical University, Yinchuan 750004, China
School of Clinical Medicine, Hainan Medical University, Haikou 571199, China

3 Department of Respiratory and Critical Care, General Hospital of Ningxia Medical University, Ningxia Medical University, Yinchuan 750004, China 